\title{
Spontaneous arteriovenous malformations in the cervical area
}

\author{
J. GREENBERG, M.D. \\ From the Department of Neurology, Episcopal Hospital, Philadelphia, Pennsylvania 19125, U.S.A.
}

SUMMARY Four patients with spontaneous arteriovenous malformations of cervical vessels have been presented. The embryology of these vessels has been discussed in order to suggest an explanation for the apparent difference in the incidence of arteriovenous malformations involving the internal carotid artery and those involving either the vertebral or the external carotid arteries. A fifth case (S.T.) is presented as a probable iatrogenic arteriovenous fistula and is to be added to the steadily growing reports of this phenomenon.

Trauma is the most common cause of arteriovenous communications between the blood vessels in the cervical area (Aronson, 1961). Iatrogenic fistulae occurring after carotid or vertebral angiography are being reported with regularity in the recent literature (Sutton, 1962). Spontaneous malformations in this area also occur. Thus far, eight cases have been reported involving the vertebral vessels (Norman, Schmidt, and Grow, 1950; Goody and Schechter, 1960; Faeth, and Dueker, 1961; Pygott and Hutton, 1964; Shumacker, Campbell, and Heimburger, 1966; Robles, 1968; Tsuji, 1968; Verbiest, 1968). 10 cases involving the external carotid system (Desprès, 1879; Beks, Bossina, and Penning, in press; Brandau, 1892; Berry, 1906; Reinhoff, 1924; Ravitch and Gaertner, 1960; Krayenbühl, 1961; Svolos, Nomikos, and Tzouliadis, 1965; Lange-Cosack,1966;Verbiest, 1968, and one common carotid jugular vein anastomosis (Callander, 1920). This report includes one patient with a spontaneous fistula of the vertebral vessels and three patients with spontaneous arteriovenous fistulae involving external carotid and branches from the subclavian artery. Because of a history of an angiogram before onset of symptoms, a fifth case (S.T.) is being reported as a probable iatrogenic vertebral arteriovenous malformation.

\section{CASE REPORTS}

CASE 1 C.S., a 5-year-old white boy, was admitted to the hospital on 15 January 1966. The chief complaint was tinnitus of progressive severity in the right ear of one year's duration. Two years before admission, he had sustained a minor injury to the posterior aspect of the right ear. Routine skull films at the time did not reveal a fracture, and there was no evidence of local deep tissue injury noted.

On the present admission, a slight prominence of the right retroauricular region was noted and a thrill and bruit were present. The bruit could be obliterated by local pressure.

The neurological examination was within normal limits. The chest radiograph revealed cardiac enlargement with prominence of the left side. There was prominence of the pulmonary vascular markings consistent with increased flow secondary to an arteriovenous shunt. A right brachial arteriogram (Fig. 1) demonstrated a large vertebral arteriovenous malformation within the high cervical area just below the foramen magnum with a major supply from the right vertebral artery and deep cervical branch of the subclavian artery. There were also branches of the thyrocervical trunk and the external occipital branch of the external carotid artery contributing to the malformation. No abnormalities were seen intracranially. A left brachial arteriogram revealed reflux from the left vertebral artery into the right vertebral artery with filling of the malformation. On 24 January 1966, the right vertebral artery was exposed. On occluding the right vertebral artery, there was no change in the thrill, which indicated that there was a massive retrograde flow from the left vertebral artery into the arteriovenous anomaly. Because of the possibility of difficulty in controlling bleeding, the surgical procedure was discontinued. The patient tolerated the procedure well.

CASE 2 L.D., a 38-year-old woman, was admitted to the hospital on 20 April 1967. Her chief complaint was a lump on the left side of her neck of two years' duration. The mass was noted to fluctuate in size, but the patient 
FIG. 1. G.S., case 1. Right brachial arteriogram demonstrating a large vertebral arteriovenous malformation.

was not aware of any pulsation. She denied neurological symptoms.

Results of the physical examination were within normal limits except for examination of the neck. Anterior to the left sternocleidomastoid muscle was a $1 \frac{1}{2} \times 1 \frac{1}{2} \mathrm{~cm}$ pulsating mass at the level of the thyroid cartilage. No thrill was felt, but a total cycle bruit was heard over the mass.

An aortic arch arteriographic study revealed a large arteriovenous malformation at the level of C6, C7 vertebrae on the left side. It was supplied by the left external carotid artery and an abnormal branch arising from the left subclavian artery. An axillary and common carotid arteriogram were performed (Fig. 2) and revealed that the malformation was supplied by a large anomalous artery arising from the left thyrocervical trunk and a hypertrophied branch from the left superior thyroid artery.

On 22 May 1967, the patient was taken to surgery and a large pulsating arteriovenous malformation was found. All the vessels leading to it were dissected free and tied. The patient tolerated the procedure well.
When seen in June 1967, she was well and had no neurological complaints.

CASE 3 J.B., a 72-year-old white woman, was admitte $\overrightarrow{9}$ to the hospital on 14 January 1966. The patient haf noted a painless lump and prominent veins in her lef. supraclavicular area, two years before admission.

Prominent veins were present on the left supra? clavicular area and over the left upper chest wall. A systolic bruit and a thrill were present in the left supra 3 clavicular area directly over the dilated veins in this region. A grade I/VI systolic ejection murmur was hear\& over the apex of the heart. Prominent veins were noted also in both feet and in both upper arms, the left greatef than the right.

A left axillary venogram (Fig. 3) revealed multip large collateral vessels on the left shoulder and uppe $\overline{5}$ upper thorax. A left subclavian arteriogram revealed aro arteriovenous fistula between the left costocervican trunk and the supraclavicular veins with moderat? tortuosity of the brachiocephalic vessels. It appeared 


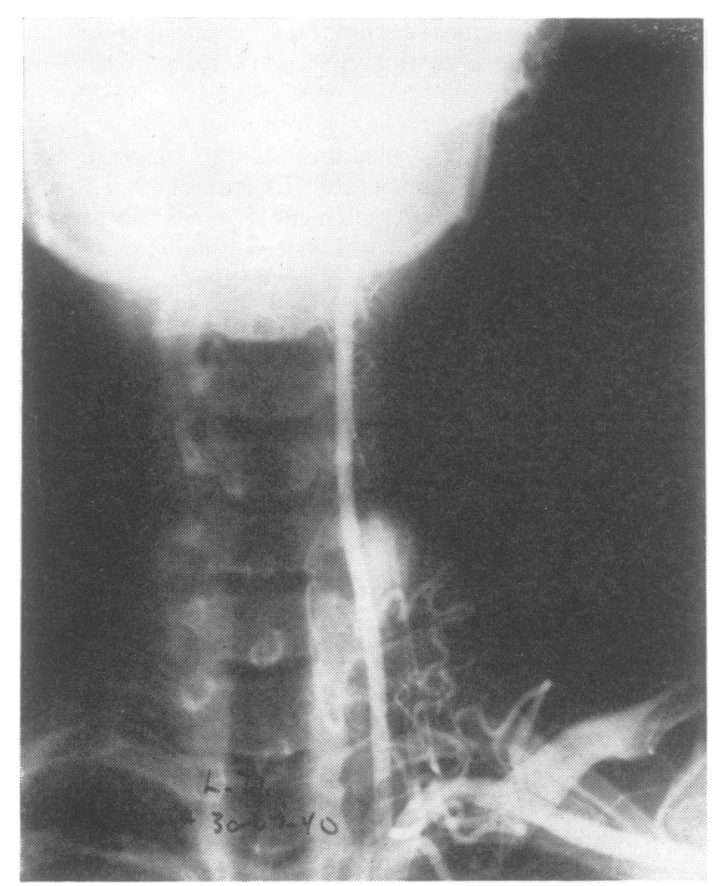

FIG. 2. L.D., case 2. Left axillary arteriogram demonstrating an arteriovenous malformation supplied by $a$ large anomalous artery arising from the left thyrocervical trunk. An arch study showed a contribution from the left external carotid artery and an abnormal branch from the left subclavian artery.

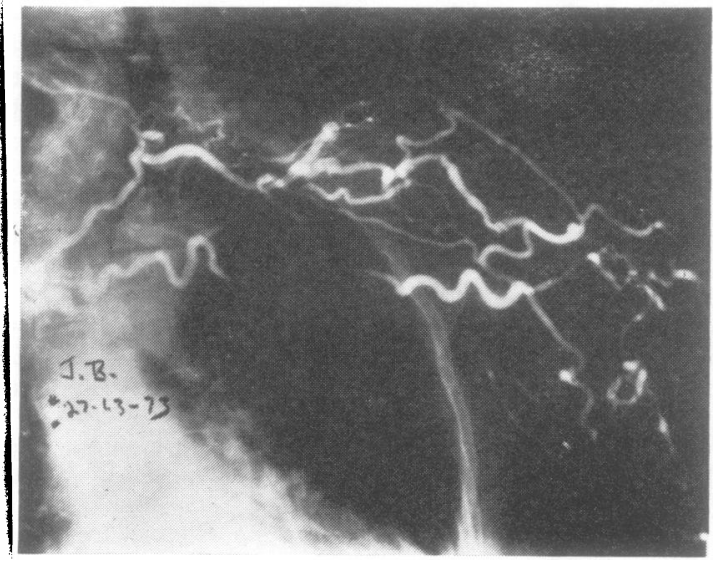

FIG. 3. J.B., case 3. Left axillary venogram revealed multiple large collaterals on the shoulder and upper thorax.

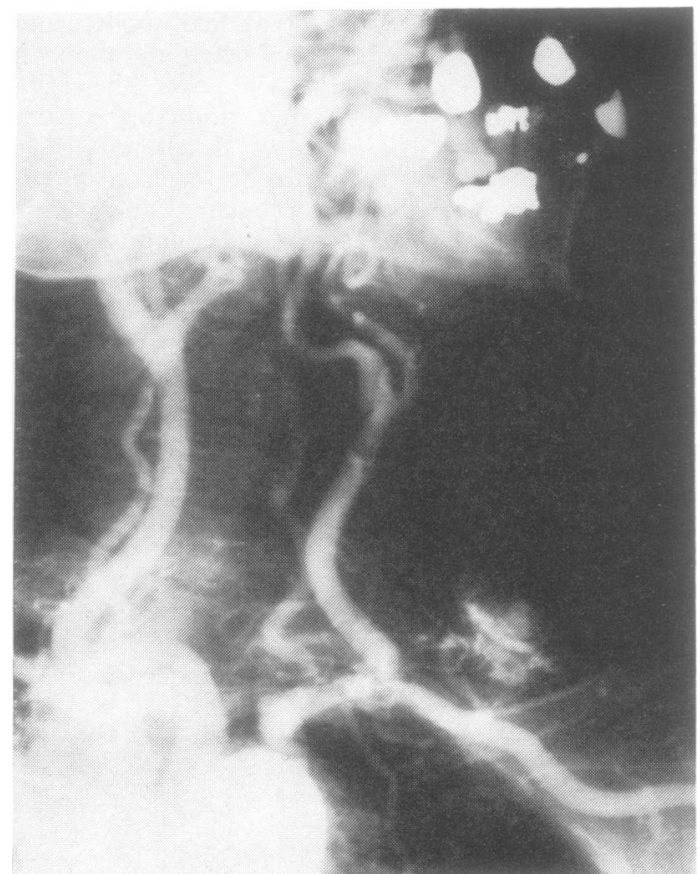

FIG. 4. J.B., case 3. Aortic arch arteriogram demonstrating an arteriovenous anomaly arising from the left thyrocervical artery.

that the thyrocervical and costocervical arteries arose from a common trunk. The costocervical artery was particularly large and quickly divided into many small vascular channels with a prominent vein which was seen to drain early. Multiple enlarged veins filled while the distal subclavian and axillary arteries were still opacified.

An aortic arch radiological study (Fig. 4) was performed which revealed no evidence of occlusive disease involving any of the major vessels of the neck. The arteriovenous fistula arising from the left thyrocervical artery was again visualized. This study revealed that the arterial supply of the arteriovenous communication came from the thyrocervical trunk primarily and fed into a large group of veins which apparently emptied into the internal jugular vein.

Some of the AV communication extended high into the neck to the level of the left mandible. There appeared to be a minor supply from the left external carotid artery but most of the arterial supply came from the left thyrocervical trunk. No other abnormalities were seen.

On 16 August 1966, the patient was taken to surgery. The skin was opened over the pulsatile mass revealing a large vascular area. The fistulous vessels were identified, ligated, and divided. The patient tolerated the procedure well and remains in good health at the present time, one year post-operatively.

CASE 4 M.S., a 21-year-old white woman, was admitted to the hospital in February 1958. Her chief complaint 
was a swollen right ear and occasional spontaneous bleeding from the right ear of one year's duration. A 'red birth-mark' had been present over the right pinna since birth. This area had gradually become swollen and developed a purplish discoloration. In October 1957, she had the first of five spontaneous bleeds from the external meatus of the right ear. Other symptoms included frontal headaches and tinnitus in the right ear in association with the pulse. The tinnitus hid become more noticeable since the onset of her first pregnancy, four months before admission.

Examination of the right ear and surrounding skin revealed a purplish discoloration. The pinna was swollen, non-tender, warm, and soft. No thrill was palpable, but a bruit was heard coincident with the pulse below the right ear. The patient was four months pregnant. The only significant laboratory study was a haemoglobin level of $7 \cdot 8 \mathrm{~g} / 100 \mathrm{ml}$.

A right carotid arteriogram demonstrated an arteriovenous fistula involving branches of the external carotid artery and a cavernous haemangioma of the right ear (Fig. 5). A radical excision of the right external carotid

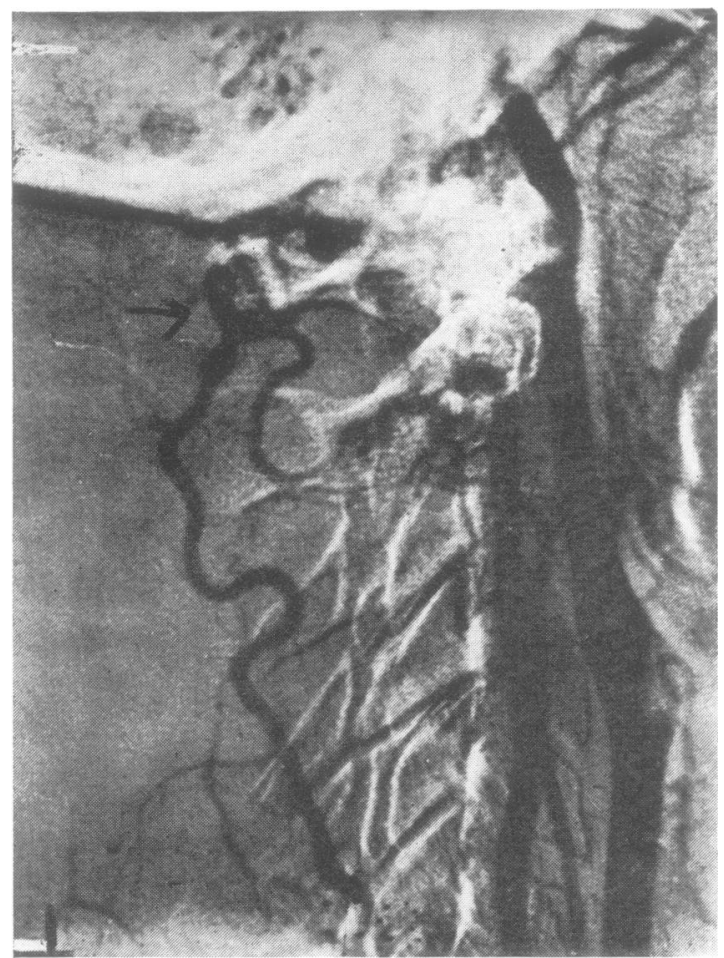

FIG. 5. M.S., Case 4. Right axillary arteriogram (subtraction film) demonstrating an arteriovenous malformation, supplied by two large branches of the right costocervical trunk. It overlies the body of the C2 vertebra and is lateral to the cervical spine. One of the vessels supplying the malformation runs parallel and anterior to the right vertebral artery and the second vessel is deep and posterior in the musculature of the neck. artery, the arteriovenous fistula, and the haemangiomata of the ear lobe were carried out on 14 December 1958 D In 1960 , a second pregnancy increased her symptoms She was readmitted to the hospital and again com? plained of spontaneous bleeding of the external ear Because of her pregnancy the patient declined any further treatment and was discharged. She was readmitteds in May 1960. A right carotid arteriogram showed n\$ major connection of the lesion with the large internaf. carotid artery. The external carotid artery was not visible having been previously resected, nor was there any con-nection between the left external carotid artery anc the lesion. On 11 May 1960 , a radical excision was performed beginning above and anterior to the eac and a large amount of haemangiomatous tissue wa․ resected. The pathology was a cirsoid aneurysm off arteriovenous fistula of the right auricular region.

CASE 5 S.T., a 23-year-old white woman, was admitted to the hospital in 1951 because of generalized headache $\vec{D}$ of recent onset. While in the hospital she abruptly developed a right hemiparesis and a right sixth andw seventh nerve paresis. Routine laboratory studies and. lumbar puncture were normal. The symptoms subsides and she was discharged.

She remained well until 1956, when she was readmitted for persistent headaches of four months' durationicu Turning her head to either side caused 'wooziness'.

A left carotid arteriogram was normal. At this tmes no bruits were heard in the neck. In 1961, she develo a 'rushing' feeling on the left side of her neck. Eer. headaches became worse and she noted intermittent blurred vision and light-headedness which were got necessarily related to neck posture. In January 196 loud bruit was noted over the left anterior part of neck during a routine physical examination. In Jüe 1965 , she was readmitted for headaches. She gave no history of previous neck trauma. A thrill was palpable over the left anterior neck. There was a grade $\mathrm{V} / \mathrm{V}$ continuous murmur heard over the entire neck, the lef occiput, and below the left clavicle. Left carotid come pression obliterated the murmur and produced para esthesiae of the left face. Compression of the right carotid artery or either subclavian artery produce no symptoms and failed to obliterate the murmur? In July 1965, a left carotid arteriogram was normal A left brachial arteriogram was performed at another hospital and revealed an arteriovenous malformation involving the left vertebral artery in the venous plexu당 at the level of the lower margin of the fifth cervicad vertebra (Fig. 6). A vertebral venous plexus was demon $\overline{\text { }}$ strated throughout its length and was greatly enlarged Deep cervical veins connected with the vertebral plexus at suggested levels (Fig. 7).

Surgery was not performed. Follow-up for nine months revealed the patient to be well other than for inter $>$ mittent headaches and light-headedness.

\section{DISCUSSION}

Arteriovenous malformations represent the per sistence of embryonic arteriovenous shunts. During 


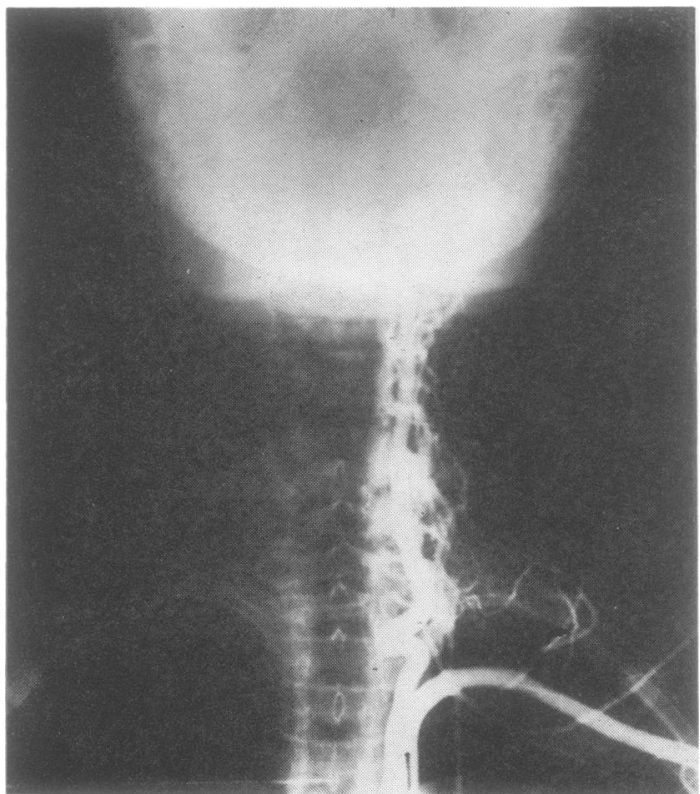

FIG. 6. S.T., case 5. Left subclavian and vertebral arteriogram by brachial artery injection. Contrast media has passed into greatly dilated vertebral veins throughout the cervical course of the vertebral artery. Note the enlarged vertebral artery.

the first period of embryological development of the blood vessels of the central nervous system, primordial endothelial blood-containing channels develop. These are neither arteries nor veins, but rather a germinal bed of endothelium. At a later stage, arteries and veins form through fusion and dissolution of a later-developed capillary mesh. An immature vascular circuit becomes functional in the 3- to 5-week embryo. The stems of origin of all cerebral arteries in the 2-month old embryo are similar to the adult. The veins reach maturity in the 3-month old embryo. However, changes in the venous system of the brain continue until birth (Congdon, 1922; Padget, 1948). It can be seen why a minor aberration occurring in early development may lead to a gross anomaly of the vascular system.

A review of the English literature has not revealed any report of spontaneous arteriovenous malformations involving the internal carotid artery. There are now reports of nine involving the vertebral artery, 13 involving the external carotid and branches of the subclavian artery, and one involving the common carotid artery and jugular vein. If the embryology of these vessels is considered, one finds interesting differences.

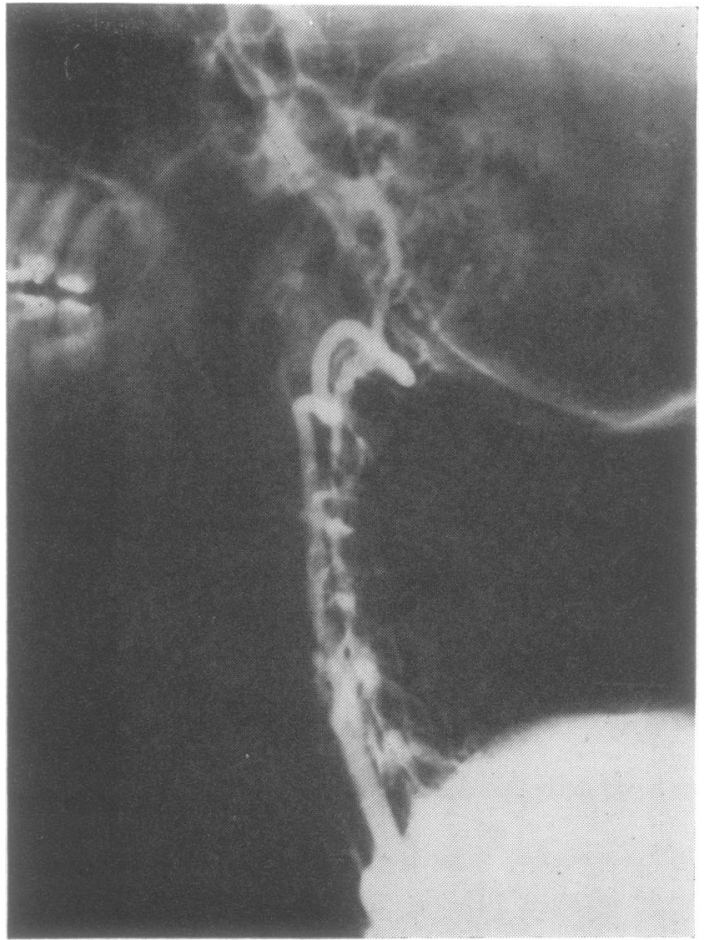

FIG. 7. S.T., case 5. Left vertebral arteriogram by brachial artery injection. The vertebral artery seen from its origin off the subclavian artery to its intracranial portion. The basilar and posterior cerebral arteries are also seen. Contrast media has passed into the vertebral venous plexus. There is an abrupt change in the calibre of the vertebral artery at the level of the lower margin of C5. It is probable that the connection is a single one at this level. Note the deep cervical veins connecting with the vertebral plexus at several levels.
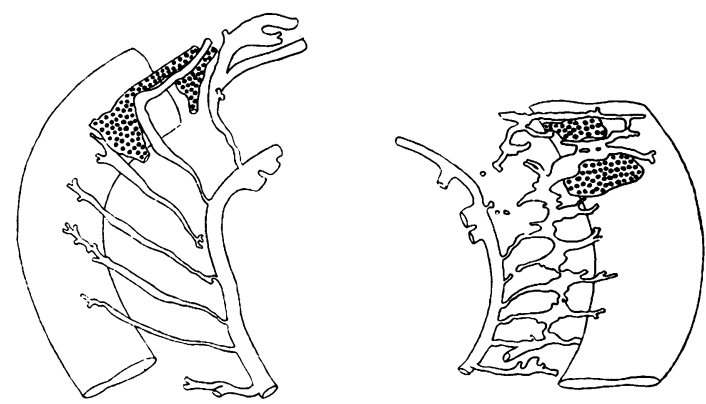

FIG. 8. Development of the vertebral arteries. Notice how the vertebral artery evolves as an anastomosis between a series of transversely running arteries (from Congdon, 1922). 


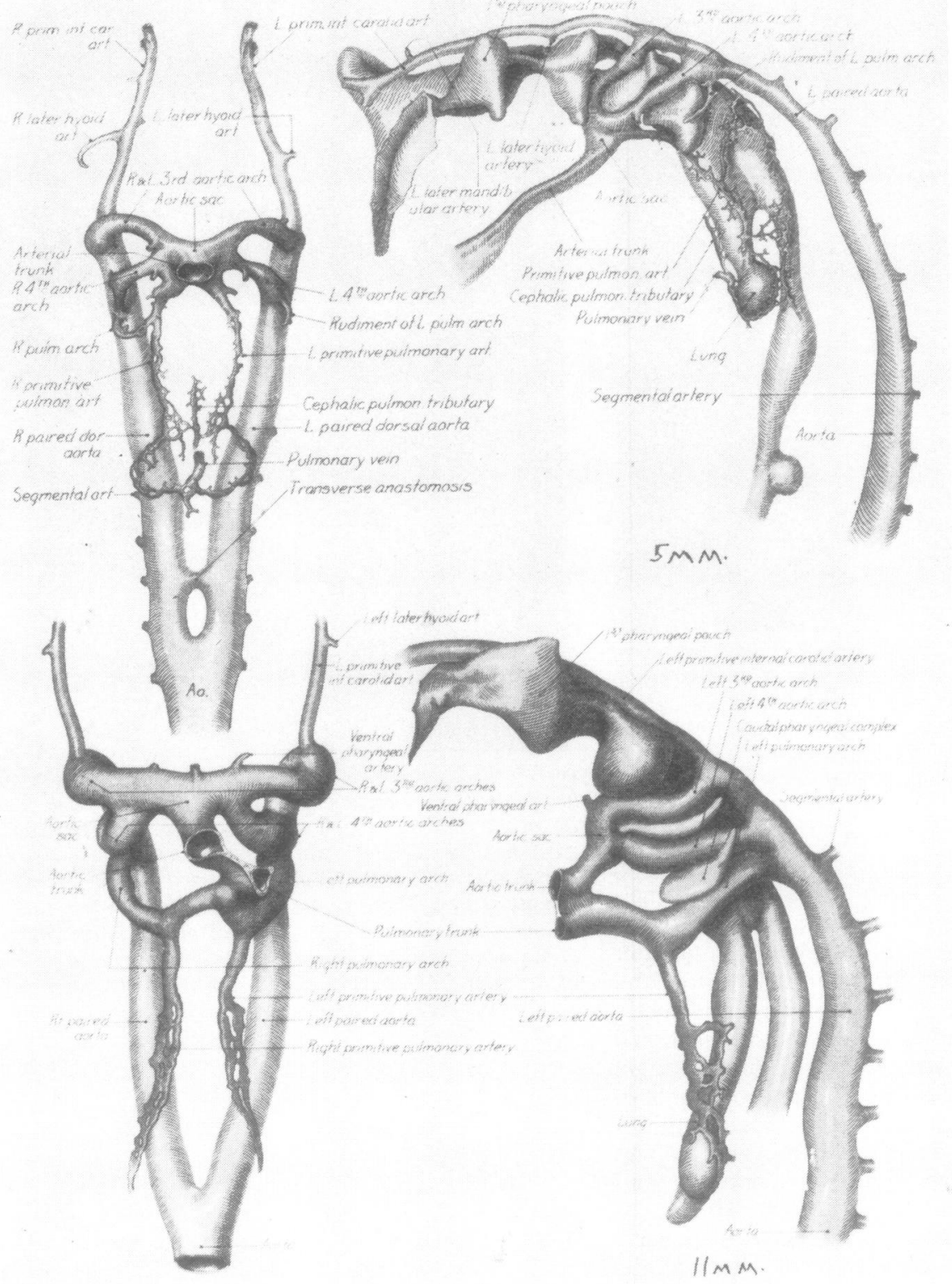

FIG. 9. Development of the carotid system (from Congdon, 1922)

The vertebral artery evolves as a longitudinal arterial channel from segments of a series of transversely running arteries and the anastomosis between them (Congdon, 1922). At its origin, there is a segment of the seventh cervical segmental artery, distal to the origin of the primitive subclavian artery. (Fig. 8). This is followed by short portions of segmental arteries as the aorta shifts downward and they are bent at each end. The gyrations that the developing vertebral artery must undergo and its tortuous course lead one to question why malformations of this vessel do not occur more often.

After the involution of the first aortic arches the terminal end of the paired aorta is recognizable as the internal carotid artery originating from the third arch (Padget, 1948) (Fig. 9). It has a more of less straight course and is not subject to the tensioris 
that the vertebral arteries experience. By 29 days (plus or minus one day) the cranial and caudal divisions of the internal carotid artery are established. The vertebral artery is formed by the 32 nd day. Perhaps these differences in development are responsible for the apparent greater tendency to arteriovenous malformations in the distribution of the vertebral vessels than in the internal carotid artery.

The external carotid system becomes definitive at age 44 days (plus or minus one day). The ventral pharyngeal artery succeeds the ventral part of the first two aortic arches and has an important role in the formation of the stapedial artery, a temporary embryonic vessel, and the external carotid artery. The proximal end of the ventral pharyngeal artery is incorporated in the stem of the external carotid artery. The stapedial artery originally pierces the stapes primordium and develops branches to the mandibular, maxillary, and supra-orbital regions. Case 4 (M.S.) could be an example of a variation at the stage of development of the external carotid and stapedial artery with her lesion at the mandible and involving at least the outer ear.

The author wishes to thank Dr. Kenneth Schulman, Dr. Mark Mishkin, and Dr. Brook Roberts for contributing cases to this report, Dr. Henry Shenkin for advice in the preparation of the manuscript, and the Carnegie Institution of Washington for permission to reproduce Figs. 8 and 9.

\section{REFERENCES}

Aronson, N. I. (1961). Traumatic arteriovenous fistula of the vertebral vessels. Angiographic demonstration and a rationale for treatment. Neurology (Minneap.), 11, 817-823.

Beks, J. W. F., Bossina, K., and Penning, L. Zbl. Neurochir. In press.

Berry, J. (1906). Large arterio-venous aneurysm of the neck treated by excision. Lancet, II, 1714-1716.

Brandau, J. W. (1892). Aneurysm by anastomosis. Nashville J. Med. Surg., 22, 193-197.

Callander, C. L. (1920). Study of arterio-venous fistula with an analysis of 447 cases. Johns Hopk. Hosp. Rep., 19, 259-358.
Congdon, E. D. (1922). Transformation of the aortic-arch system during the development of the human embryo, Contrib. Embryol. Carneg. Inst., 14, 47-110.

Desprès, M. (1879). Tumeur veineuse du plancher de la bouche. Anévrysme artérioveineux. Ligature des deux artères linguales. Bull. Soc. Chir. Paris, 5, 794-800.

Faeth, W. H., and Dueker, H. W. (1961). Arteriovenous vascular malformation of the cervical portion of the vertebral artery. Neurology (Minneap.), 11, 492-493.

Goody, W., and Schechter, M. M. (1960). Spontaneous arterio-venous fistula of the vertebral artery. Brit. $J$. Radiol., 33, 709-711.

Krayenbühl, H. (1961). Vaskuläre Krankheiten im extrakraniellen Karotis-Vertebralis-Bereich als Ursache intrakranieller Geräusche. Münch. med. Wschr., 103, 2185-2187.

Lange-Cosack, H. (1966). Anatomie und Klinik der Gefässmissbildungen des Gehirns und seiner Häute. Handbuch der Neurochirurgie. Vol. 4, part 2, pp. 1-45. Edited by H. Olivecrona and W. Tönnis. SpringerBerlin.

Norman, J. A., Schmidt, K. W., and Grow, J. B. (1950). Congenital arterio-venous fistula of the cervical vertebral vessels with heart failure in an infant. J. Pediat., 36, 598-604.

Padget D. H. (1948). The development of the cranial arteries in the human embryo. Contrib. Embryol. Carneg. Inst., 32, 206-261.

Pygott, F., and Hutton, C. F. (1964). Angioma of the neck with intra-spinal extension. Brit. J. Radiol., 37, 72-73.

Reinhoff, W. F., Jun. (1924). Congenital arteriovenous fistula. An embryological study, with the report of a case. Bull. Johns Hopk. Hosp., 35, 271-284.

Ravitch, M. M., and Gaertner, R. A. (1960). Congenital arteriovenous fistula in the neck-48 year follow-up of a patient operated upon by Dr. Halsted in 1911 . Bull. Johns Hopk. Hosp., 107, 31-56.

Robles, J. (1968). Congenital arteriovenous malformation of the vertebral vessels in the neck. Case report. $J$. Neurosurg., 29, 206-208.

Shumacker, H. B., Campbell, R. L., and Heimburger, R. F. (1966). Operative treatment of vertebral arteriovenous fistulas. J. Trauma, 6, 3-19.

Sutton, D. (1962). Arteriography, p. 54. E. \& S. Livingstone: Edinburgh.

Svolos, D., Nomikos, N., and Tzouliadis, V. (1965). Congenital arterio-venous aneurysm in the neck. $J$. Neurosurg., 23, 68-71.

Tsuji, H. K. (1968). Unpublished-mentioned in J. thor. cardiovasc. Surg., 55, 746-753.

Verbiest, H. (1968). Extracranial and cervical arteriovenous aneurysms of the carotid and vertebral arteries, Report of a series of 12 personal cases. Johns Hopk. Med. J., 122, 350-357. 